\title{
Feedback control of plasma etching reactors for improved etching uniformity
}

\author{
Antonios Armaou*, James Baker, Panagiotis D. Christofides \\ Department of Chemical Engineering, University of California, Los Angeles, CA 90095, USA
}

\begin{abstract}
This work focuses on the design and implementation of a feedback control system on a parallel electrode plasma etching (PE) process with showerhead arrangement used to etch a $500 \mathrm{~A}$ amorphous silicon thin film on a 4 in wafer. The feedback control system consists of three spatially distributed proportional integral controllers that use measurements of the etching rate at three locations across the wafer to manipulate the inlet concentration of carbon tetrafluoride in the showerhead. The controller is implemented on a detailed fundamental model of the process, which accounts for diffusive and convective mass transfer, bulk and surface reactions, and non-uniform fluid flow and plasma electron density profiles, and is shown to reduce the etching rate nonuniformity from $30.2 \%$ to $3.8 \%$. (C) 2001 Elsevier Science Ltd. All rights reserved.

Keywords: Plasma etching; Distributed control; Spatial uniformity control
\end{abstract}

\section{Introduction}

Single-wafer plasma etching (PE) is an important process used in semiconductor manufacturing to selectively remove areas of thin films of compounds (e.g. amorphous silicon, silicon nitride, etc.) which are placed on substrates. In a PE process, the activation of the gases is achieved through impact with electrons and leads to the reacting species. The main advantages of plasma-assisted etching include directional selectivity of the reactions that take place on the surface of the wafer (etch anisotropy), reduced processing time and elimination of wet chemicals used in traditional etching processes. Even though the advantages of plasma etching are well known, a major issue which limits the widespread use of the PE process is the significant spatial nonuniformity of the etching rate. Radially nonuniform etching rates result in nonuniform film thickness, which adversely affects the subsequent processing steps, and ultimately, product yield. This problem is usually addressed with over-etching, leading to: (a) higher than the necessary time for the specific process step, and (b) degradation of the structures, resulting also in lower product yield. The design

\footnotetext{
* Corresponding author.
}

and operation of PE processes that achieve the desired performance specifications require the derivation of accurate mathematical models that can be used to design optimal reactor, flow arrangements and test the performance of different control systems.

Key issue in the development of high-fidelity models is the understanding and characterization of the physicochemical phenomena taking place in PE processes. In this direction, research efforts have been focused on the characterization of plasma chemistry (e.g. Flamm, Donnelly, \& Ibbotson, 1983; Plumb \& Ryan, 1986), the experimental measurement of electron density profiles in plasma reactors (e.g. Belenguer \& Boeuf, 1990), and the identification of surface reactions and their mechanisms (e.g. Flamm, Donnelly, \& Mucha, 1981; Plumb \& Ryan, 1986). The improved understanding of plasma properties has led to the development of accurate mathematical models for various plasma etching processes which can be used to compute reactor configurations that reduce the etching nonuniformity (e.g. Park \& Economou, 1990; Park \& Economou, 1991; Bushman, Edgar, \& Trachtenberg, 1997; Venkatesan, Trachtenberg, \& Edgar, 1987). Such studies have revealed that the use of a showerhead arrangement to introduce the precursor gas into the reaction chamber, significantly reduces the radial etching nonuniformity. Previous works on control of plasma etching processes include radical concentration control 
(McLaughlin, Butler, Edgar, \& Trachtenberg, 1991; Butler, McLaughlin, Edgar, \& Trachtenberg, 1991; Mozumder, Saxena, \& Collins, 1994), and nonuniformity control using feedback control schemes (Hankinson, Vincent, Irani, \& Khargonekar, 1997; Van Antwerp, Braatz, \& Sahinidis, 1997).

In this work, we consider a parallel electrode PE process with showerhead arrangement used to etch a $500 \AA$ amorphous silicon film on a 4 in wafer. The objective is to develop a detailed fundamental model for this process, able to predict the etching rate profile across the radius of the wafer, and a feedback control strategy that can substantially reduce the etching rate radial nonuniformity. Initially, a two-dimensional dynamic model is developed that accounts for diffusive and convective mass transfer, bulk etching reactions, and nonuniform fluid flow and plasma electron density profiles. The model predicts that the radial etching rate nonuniformity is $30.2 \%$. Then, a feedback control system is designed and implemented on the process to reduce this nonuniformity. The control system consists of three spatially distributed proportional integral controllers that use measurements of the etching rate at three locations across the wafer, to manipulate the inlet concentration of carbon tetrafluoride in the showerhead and achieve a uniform etching rate across the radius of the wafer. The implementation of the proposed control system is shown to reduce the etching rate nonuniformity to $3.8 \%$.

\section{Process description and modeling}

We consider a parallel electrode PE process with showerhead arrangement (Park \& Economou, 1991) shown in Fig. 1. The objective of the process is to etch a $500 \AA$ amorphous silicon thin film placed on a 4 in wafer, which is located on the top of the lower electrode. The reactor is fed through the showerhead by a gas stream including carbon tetrafluoride, $\mathrm{CF}_{4}$, and inert gases (typically $\mathrm{He}$ or Ar). The flow rate and composition of the influent gas stream are assumed to be uniform throughout the showerhead. A radio frequency $(\mathrm{RF})$ power source is used to generate the plasma (i.e., chemically reactive mixture of ions, electrons and radicals) from $\mathrm{CF}_{4}$. The radicals are then transported via convection and diffusion to the surface of the wafer where they react towards $\mathrm{SiF}_{4}$, etching the amorphous silicon. The plasma species that do not react with silicon, the etching products, and the unreacted $\mathrm{CF}_{4}$ are pumped radially outwards. The reactor is assumed to be at 0.5 Torr pressure and $325 \mathrm{~K}$ temperature, while a $13.56 \mathrm{MHz}$ frequency alternate voltage is applied to the electrodes generating a glow discharge. The wafer is assumed to be at a constant temperature of $400 \mathrm{~K}$ which is accomplished by a heating coil situated underneath the lower electrode. In the remainder of this section, we develop a fundamental model for the PE reactor of Fig. 1.

\subsection{Flow field}

The gas flow inside the reactor resembles the threedimensional axisymmetric stagnation flow. Under the standard assumptions: (a) treating plasma as a continuum medium, (b) constant physical properties of the gas, (C) negligible volume change of the reacting gases, and (d) azimuthal reactor symmetry, the flow field can be described by using the Navier-Stokes and continuity equations. The feed enters the chamber through the showerhead upper electrode at a constant velocity, $v_{w}$, which is assumed to be radially uniform and is calculated from the equation: $v_{w}=Q\left(T_{r}, P\right) /\left(N_{h} \pi r_{h}^{2}\right)$, where $Q\left(T_{r}, P\right)$ is the volumetric rate of the precursor gas at the operating conditions of the reactor, $T_{r}$ is the reactor temperature, $P$ is the pressure, $N_{h}$ is the number of holes on the top electrode, and $r_{h}$ is the radius of each hole. Defining the wall Reynolds number as $\operatorname{Re}_{w}=\left(v_{w} L \rho\right) / 4 \mu$, where $L$ is the height of the reactor (i.e., distance between the two electrodes), $\rho$ is the (constant) density of the plasma, and $\mu$ is the (constant) viscosity of the plasma, the operating conditions of Table 1 correspond to $R e_{w}=0.61<1$. For this value of Reynold's number, the following steady-state approximate analytic solution of the Navier-Stokes and continuity equations can be computed

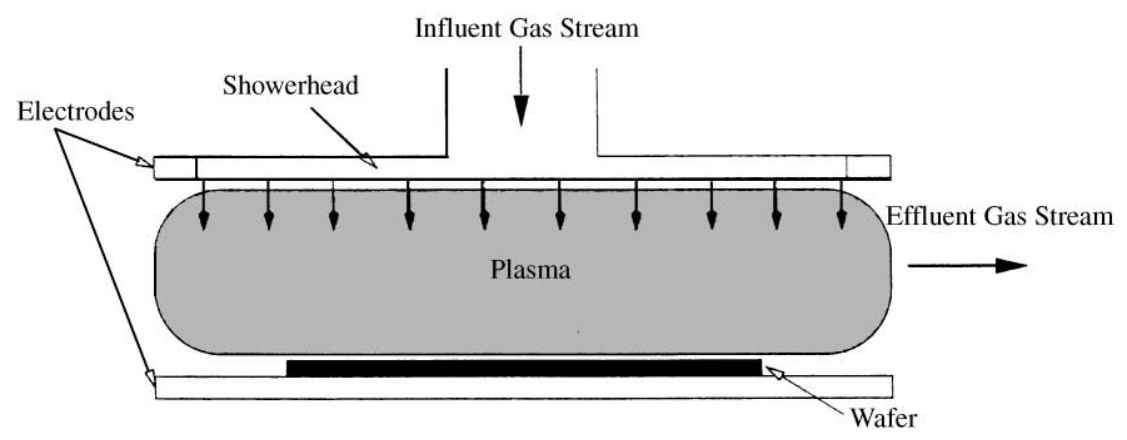

Fig. 1. Cylindrical parallel electrode plasma etch reactor with showerhead configuration. 
Table 1

Process parameters

$$
\begin{aligned}
& L=3.6 \mathrm{~cm} \\
& r_{t}=8.0 \mathrm{~cm} \\
& r_{w}=5.08 \mathrm{~cm} \\
& Q=50.0 \mathrm{scm}^{3} \mathrm{~min}^{-1} \\
& N_{h}=350 \\
& r_{h}=0.15 \mathrm{~cm} \\
& P=0.5 \mathrm{Torr} \\
& T_{r}=325 \mathrm{~K} \\
& T_{w}=400 \mathrm{~K} \\
& n e_{o}=1.5 \times 10^{10} \mathrm{~cm}^{-3} \\
& \mu=1.7756 \times 10^{-7} \mathrm{~kg} \mathrm{~s}^{-1} \mathrm{~cm}^{-1} \\
& \rho=2.171 \times 10^{-9} \mathrm{~kg} \mathrm{~cm}^{-3} \\
& \rho_{\mathrm{Si}}=8.292 \times 10^{-10} \mathrm{~mol} \mathrm{~A}^{-1} \mathrm{~cm}^{-2}
\end{aligned}
$$

(for a more detailed analysis the reader may refer to Armaou \& Christofides (1999) and Economou, Park, $\&$ Williams (1989), under the assumption that the velocity in the $z$-direction, $v_{z}$, is only a function of $z$ :

$$
\begin{aligned}
v_{r}(z, r)= & \frac{v_{w}}{L} r\left[-\frac{3}{4} \psi^{2}+\frac{3}{4}+R e_{w}\left(-\frac{1}{160} \psi^{6}+\frac{3}{32} \psi^{4}\right.\right. \\
& \left.\left.+\frac{1}{4} \psi^{3}-\frac{117}{1120} \psi^{2}-\frac{1}{4} \psi+\frac{19}{1120}\right)\right], \\
v_{z}(z)= & -v_{w}\left[-\frac{1}{4} \psi^{3}+\frac{3}{4} \psi+\frac{1}{2}\right. \\
& +\operatorname{Re}_{w}\left(-\frac{1}{1120} \psi^{7}+\frac{3}{160} \psi^{5}+\frac{1}{16} \psi^{4}\right. \\
& \left.\left.-\frac{39}{1120} \psi^{3}-\frac{1}{8} \psi^{2}+\frac{19}{1120} \psi+\frac{1}{16}\right)\right],
\end{aligned}
$$

where $\psi=2 z / L-1, v_{r}$ is the velocity in the $r$-direction, $v_{z}$ is the velocity in the $z$-direction, $r \in\left[0, r_{t}\right]$ is the radial coordinate, $r_{t}$ is the radius of the reactor, and $z \in[0, L]$ is the axial coordinate. The above analytic expressions for $v_{r}(z, r)$ and $v_{z}(z)$ were employed in the simulations of Sections 3 and 4. Referring to the flow field, we also note that the axisymmetric stagnation flow inside the reactor implies that the Reynolds number computed with respect to all the values of the velocity vector throughout the entire flow field does not vary significantly and is everywhere less than 1. This in turn means that there are no sharp velocity gradients in the flow field close to the showerhead which could lead to jetting effects and flow instabilities (this is also the case for the closed-loop system as discussed in Section 4).

\subsection{Electron density profile}

For 0.5 Torr pressure and $13.56 \mathrm{MHz}$ frequency alternate voltage, the secondary electron emission is negli- gible and the glow is mainly sustained by bulk ionization. Therefore, the steady-state electron density profile is computed by assuming diffusion-controlled discharge in a cylindrical container and is given by the following formula (Park \& Economou, 1991):

$$
n_{e}(r, z)=n_{e o} J_{0}\left(2.405 \frac{r}{r_{t}}\right) \sin \left(\frac{\pi z}{L}\right),
$$

where $n_{e}(r, z)$ is the electron density, $n_{e o}$ is the maximum electron density in the reactor $\left(n_{e o}\right.$ depends on the power dissipated in the plasma, the plasma volume and the effective electric field; the value of $n_{e o}$ used in our calculations was computed in Ryan \& Plumb (1986) based on experimental data), $J_{0}$ is the zero-order Bessel function of the first kind and $r_{t}$ is the radius of the reactor. From Eq. (2), it is clear that the electron density profile attains its maximum value in the center of the reactor (i.e. $r=0$, $z=L / 2)$.

\subsection{Reactions and mass transport}

Regarding the reactions that take place on the bulk of the plasma, we assume that $\mathrm{CF}_{4}$ enters the reactor and dissociates according to the following reaction scheme (Flamm et al., 1983; Edelson \& Flamm, 1984):

$$
\begin{aligned}
& e^{-}+\mathrm{CF}_{4} \rightarrow \mathrm{CF}_{3}+\mathrm{F}+e^{-}, \\
& e^{-}+\mathrm{CF}_{4} \rightarrow \mathrm{CF}_{2}+2 \mathrm{~F}+e^{-}, \\
& \mathrm{F}+\mathrm{CF}_{2} \rightarrow \mathrm{CF}_{3}, \\
& \mathrm{~F}+\mathrm{CF}_{3} \rightarrow \mathrm{CF}_{4}, \\
& \mathrm{CF}_{3}+\mathrm{CF}_{3} \rightarrow \mathrm{C}_{2} \mathrm{~F}_{6},
\end{aligned}
$$

where $e^{-}$is the electron, $\mathrm{CF}_{3}$ is trifluoromethyl radical, $\mathrm{CF}_{2}$ is difluoromethylene, $\mathrm{F}$ is the fluorine radical and $\mathrm{C}_{2} \mathrm{~F}_{6}$ is hexafluoroethane. According to this reaction scheme, $\mathrm{CF}_{4}$ initially dissociates due to impact with electrons forming difluoromethylene, trifluoromethyl radical and atomic fluorine; this stage is described by the reactions: $e^{-}+\mathrm{CF}_{4} \rightarrow \mathrm{CF}_{3}+\mathrm{F}+e^{-}, \quad e^{-}+\mathrm{CF}_{4} \rightarrow$ $\mathrm{CF}_{2}+2 \mathrm{~F}+e^{-}$. The fluorine radical $\mathrm{F}$ then reacts with $\mathrm{CF}_{2}$ and subsequently with $\mathrm{CF}_{3}$ according to the recombination reactions $\mathrm{F}+\mathrm{CF}_{2} \rightarrow \mathrm{CF}_{3}, \mathrm{~F}+\mathrm{CF}_{3} \rightarrow \mathrm{CF}_{4}$ forming $\mathrm{CF}_{4} \cdot \mathrm{CF}_{3}$ also recombines to form $\mathrm{C}_{2} \mathrm{~F}_{6}$, according to the reaction $\mathrm{CF}_{3}+\mathrm{CF}_{3} \rightarrow \mathrm{C}_{2} \mathrm{~F}_{6}$. Assuming that the effective reaction rate of the reactions of Eq. (3) can be expressed as a product of the dissociation constants, electron density and reactant species concentrations (Edelson \& Flamm, 1984; Park \& Economou, 1991), the following expressions can be written for the rate of change of the concentration, $r_{x, i}$, of the species $\mathrm{CF}_{4}, \mathrm{CF}_{2}, \mathrm{CF}_{3}, \mathrm{~F}$ due to consumption and production 
according to the reactions of Eq. (3):

$r_{x, 1}=-k_{1} n_{e} c_{1}-k_{2} n_{e} c_{1}+k_{4} c_{3} c_{4}$,

$r_{x, 2}=k_{2} n_{e} c_{1}-k_{3} c_{2} c_{4}$,

$r_{x, 3}=k_{1} n_{e} c_{1}+k_{3} c_{2} c_{4}-k_{4} c_{3} c_{4}-k_{5} c_{3} c_{3}$,

$r_{x, 4}=k_{1} n_{e} c_{1}+2 k_{2} n_{e} c_{1}-k_{3} c_{2} c_{4}-k_{4} c_{3} c_{4}$,

where the subscripts $i=1,2,3,4$ correspond to carbon tetrafluoride $\mathrm{CF}_{4}$, difluoromethylene $\mathrm{CF}_{2}$, trifluoromethyl radical $\mathrm{CF}_{3}$, and fluorine radical $\mathrm{F}$ respectively, $c_{i}$ is the concentration of each species, and $k_{j}$, $j=1,2,3,4,5$ is the reaction rate constant for each reaction in the scheme of Eq. (3).

The radicals $\mathrm{CF}_{3}$ and $\mathrm{F}$ that are produced in the bulk of the plasma are transported to the wafer surface where they react with amorphous silicon $\mathrm{Si}$ according to (Flamm et al. (1981) and Ryan \& Plumb (1986)):

$\mathrm{CF}_{3}(g)+\mathrm{Si}(s) \rightarrow \mathrm{CF}_{3}(s)$,

$\mathrm{F}(g)+\mathrm{CF}_{3}(s) \rightarrow \mathrm{CF}_{4}(g)$,

$\mathrm{CF}_{3}(g)+\mathrm{CF}_{3}(s) \rightarrow \mathrm{C}_{2} \mathrm{~F}_{6}(g)$,

$4 \mathrm{~F}(g)+\mathrm{Si}(s) \rightarrow \mathrm{SiF}_{4}(g)$,

where $\mathrm{SiF}_{4}$ is tetrafluorosilane, $(g)$ denotes the gas phase and $(s)$ denotes the surface bound species. Referring to the above scheme, note that only the last reaction leads to the etching of amorphous silicon. Based on this reaction scheme, the rate of change of the concentration on the wafer surface, $r_{s, i}$, of the species $\mathrm{CF}_{4}, \mathrm{CF}_{2}, \mathrm{CF}_{3}, \mathrm{~F}$ can be expressed as

$r_{s, 1}=k_{s 2} c_{4} \theta, \quad r_{s, 2}=0$,

$r_{s, 3}=-k_{s 3} c_{3} \theta-k_{s 1} c_{3}(1-\theta)$,

$r_{s, 4}=-k_{\mathrm{etch}}(1-\theta)-k_{s 2} c_{4} \theta$,

where $c_{i}$ is the concentration of each species in the gas phase, $k_{s j}, j=1,2,3$ is the reaction rate constant for the first three reactions in the scheme of Eq. (5), respectively, and $k_{\text {etch }}$ is the reaction rate constant of the etching reaction (fourth reaction). $\theta$ denotes the surface coverage of $\mathrm{CF}_{3}$ and is given from the following expression:

$\theta=\frac{k_{s 1} c_{3(g)}}{\left(k_{s 3}+k_{s 1}\right) c_{3(g)}+k_{s 2} c_{4(g)}}$,

under the assumption of equilibrium for the surface reactions. Since we assume isothermal operation for both the reactor and wafer surface, the reaction rate constants of the bulk and surface reactions are taken to be constant.

Applying dynamic material balances to the process and accounting for diffusive and convective mass transfer, and bulk and surface reactions, a mathematical model that describes the spatio-temporal evolution of the
Table 2

Bulk reaction rate constants

\begin{tabular}{lll}
\hline Reaction & Rate constant & Units \\
\hline$e^{-}+\mathrm{CF}_{4} \rightarrow \mathrm{CF}_{3}+\mathrm{F}+e^{-}$ & $6.00 \times 10^{-10}$ & $\mathrm{~s}^{-1} \mathrm{~cm}^{3}$ \\
$e^{-}+\mathrm{CF}_{4} \rightarrow \mathrm{CF}_{2}+2 \mathrm{~F}+e^{-}$ & $1.40 \times 10^{-9}$ & $\mathrm{~s}^{-1} \mathrm{~cm}^{3}$ \\
$\mathrm{~F}+\mathrm{CF}_{2} \rightarrow \mathrm{CF}_{3}$ & $2.53 \times 10^{11}$ & $\mathrm{~s}^{-1} \mathrm{~mol}^{-1} \mathrm{~cm}^{3}$ \\
$\mathrm{~F}+\mathrm{CF}_{3} \rightarrow \mathrm{CF}_{4}$ & $7.83 \times 10^{12}$ & $\mathrm{~s}^{-1} \mathrm{~mol}^{-1} \mathrm{~cm}^{3}$ \\
$\mathrm{CF}_{3}+\mathrm{CF}_{3} \rightarrow \mathrm{C}_{2} \mathrm{~F}_{6}$ & $4.82 \times 10^{12}$ & $\mathrm{~s}^{-1} \mathrm{~mol}^{-1} \mathrm{~cm}^{3}$ \\
\hline
\end{tabular}

Table 3

Surface reaction rate constants

\begin{tabular}{lll}
\hline Reaction & Rate constant & Units \\
\hline $\mathrm{CF}_{3}(g)+\mathrm{Si}(s) \rightarrow \mathrm{CF}_{3}(s)$ & 17.52 & $\mathrm{~s}^{-1} \mathrm{~cm}^{3}$ \\
$\mathrm{~F}(g)+\mathrm{CF}_{3}(s) \rightarrow \mathrm{CF}_{4}(g)$ & 85.80 & $\mathrm{~s}^{-1} \mathrm{~cm}^{3}$ \\
$\mathrm{CF}_{3}(g)+\mathrm{CF}_{3}(s) \rightarrow \mathrm{C}_{2} \mathrm{~F}_{6}(g)$ & 08.76 & $\mathrm{~s}^{-1} \mathrm{~cm}^{3}$ \\
$4 \mathrm{~F}(g)+\mathrm{Si}(s) \rightarrow \mathrm{SiF}_{4}(g)$ & 85.24 & $\mathrm{~s}^{-1} \mathrm{~cm}^{3}$
\end{tabular}

concentration of the species, $\mathrm{CF}_{4}, \mathrm{CF}_{2}, \mathrm{CF}_{3}$ and $\mathrm{F}$, throughout the reactor is obtained. The model consists of four nonlinear parabolic partial differential equations and can be written in the following compact form:

$$
\begin{aligned}
\frac{\partial c_{i}}{\partial t}= & D_{i}\left(\frac{\partial^{2} c_{i}}{\partial r^{2}}+\frac{1}{r} \frac{\partial c_{i}}{\partial r}+\frac{\partial^{2} c_{i}}{\partial z^{2}}\right)-v_{r} \frac{\partial c_{i}}{\partial r}-v_{z} \frac{\partial c_{i}}{\partial z} \\
& +r_{x, i}\left(n_{e}, c_{1}, c_{2}, c_{3}, c_{4}\right), \quad i=1, \ldots, 4
\end{aligned}
$$

subject to the boundary conditions:

$\frac{\partial c_{i}}{\partial r}(t, 0, z)=0, \quad \frac{\partial c_{i}}{\partial r}\left(t, r_{t}, z\right)=0, \quad i=1, \ldots, 4$,

$D_{i} \frac{\partial c_{i}}{\partial z}(t, r, 0)=r_{s, i}, \quad 0 \leqslant r \leqslant r_{w}$,

$\frac{\partial c_{i}}{\partial z}(t, r, 0)=0, \quad r_{w}<r \leqslant r_{t}$,

$c_{i}(t, r, L)=c_{w, i}(t, r), \quad i=1, \ldots, 4$

and the initial conditions

$c(0, r, z)=c_{i}^{0}(r, z), \quad i=1, \ldots, 4$,

where $D_{i}$ is the diffusion coefficient of the $i$ th species, $r_{w}$ is the radius of the wafer, $c_{w, i}(t)$ is the concentration of the specific species at the feed and $c_{i}^{0}(r, z)$ is the concentration of the species initially in the chamber. The boundary conditions outside the wafer surface (Eq. (10)) result from the fact that $\mathrm{CF}_{4}, \mathrm{CF}_{2}, \mathrm{CF}_{3}, \mathrm{~F}$ do not react with the walls of the reactor. The etching rate of amorphous silicon on the wafer is calculated from the following equation (Park \& Economou, 1991):

$R_{\mathrm{etch}}(t, r)=0.25 \rho_{\mathrm{Si}} k_{\mathrm{etch}} c_{4}(t, r, 0)(1-\theta(t, r))$,

where $\rho_{\mathrm{Si}}$ is the density of $a$-Si and $\theta$ is given from Eq. (7).

The values of the operating conditions and the reactor parameters are given in Table 1, the values of the reaction rate constants are presented in Tables 2 and 3, and 
Table 4

Physico-chemical properties of plasma species

\begin{tabular}{llllll}
\hline Properties & $\mathrm{CF}_{4}$ & $\mathrm{CF}_{2}$ & $\mathrm{CF}_{3}$ & $\mathrm{~F}$ & Units \\
\hline$D_{i}$ & 110.85 & 183.97 & 125.38 & 405.44 & $\mathrm{~cm}^{2} \mathrm{~s}^{-1}$ \\
$c_{i}^{0} / c_{t}$ & 1.0 & 0.0 & 0.0 & 0.0 & \\
$c_{w, i} / c_{t}$ & 1.0 & 0.0 & 0.0 & 0.0 & \\
\hline
\end{tabular}

finally, the values of physico-chemical properties of the species are tabulated in Table 4. We note that: (a) the physical properties of the plasma (e.g., viscosity) were computed by assuming that the plasma consists of pure carbon tetrafluoride (See also (Reid, Prausnitz, \& Poling, 1987)), and (b) the density of the mixture was calculated from the ideal gas law.

\section{Open-loop simulation results}

The mathematical model of the PE reactor consisting of the four unsteady-state two-dimensional diffusionconvection-reaction equations (Eq. (8)), the velocity (Eq. (1)) and electron density (Eq. (2)) profiles, was solved by using numerical techniques. Following Armaou and Christofides (1999), the finite-difference method was initially used to discretize the spatial derivatives of Eq. (8) in the $r$ - and $z$-directions ( 75 and 75 discretization points were used in the $r$ - and $z$-direction, respectively; it was

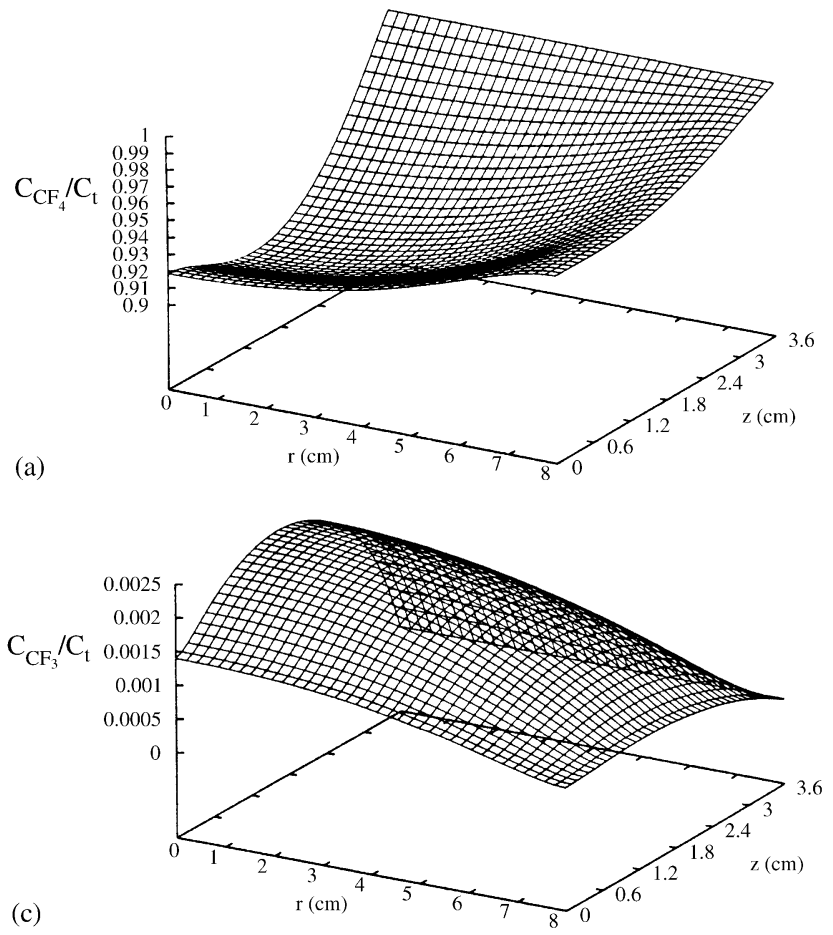

verified that further increase in the number of discretization points in both $r$ - and $z$-directions results in insignificant improvements in the accuracy of the computed solution). The time-integration of the resulting large $(75 \times 75)$ set of ordinary differential equations was performed by utilizing the alternate direction implicit (ADI) method. In all the simulation runs, the reactor is initially assumed to be filled with pure carbon tetrafluoride. Fig. 2a shows the profile of the steady-state dimensionless concentration of carbon tetrafluoride, $\mathrm{CF}_{4}$, inside the reactor (in the $r$-axis: $0 \mathrm{~cm}$ is the center of the wafer, $5.08 \mathrm{~cm}$ is the edge of the wafer and $8.0 \mathrm{~cm}$ is the edge of the reactor; in the $z$-axis: $0 \mathrm{~cm}$ is the wafer surface and $3.6 \mathrm{~cm}$ is the showerhead). We observe that the concentration of $\mathrm{CF}_{4}$ is lesser towards the center of the reactor. This is because $\mathrm{CF}_{4}$ is mainly consumed by the dissociation reactions, whose rate is larger at the center of the reactor owing to the maximum electron density profile there. Figs. $2 \mathrm{~b}$ and $\mathrm{c}$ show the profiles of the steady-state concentration of difluoromethylene, $\mathrm{CF}_{2}$, and trifluoromethyl radical, $\mathrm{CF}_{3}$, respectively. The concentration of $\mathrm{CF}_{2}$ is much higher than the concentration of $\mathrm{CF}_{3}$ due to the higher reactivity of $\mathrm{CF}_{3}$ in the bulk of the plasma and wafer surface and higher dissociation rate of $\mathrm{CF}_{4}$ towards $\mathrm{CF}_{2}$. As expected, the maximum steadystate concentration for both $\mathrm{CF}_{2}$ and $\mathrm{CF}_{3}$ is located at the center of the reactor due to the maximum electron density in this location.

The nonuniform concentration of $\mathrm{CF}_{3}$ and $\mathrm{F}$ on the wafer surface, together with the fact that the etching rate (b)
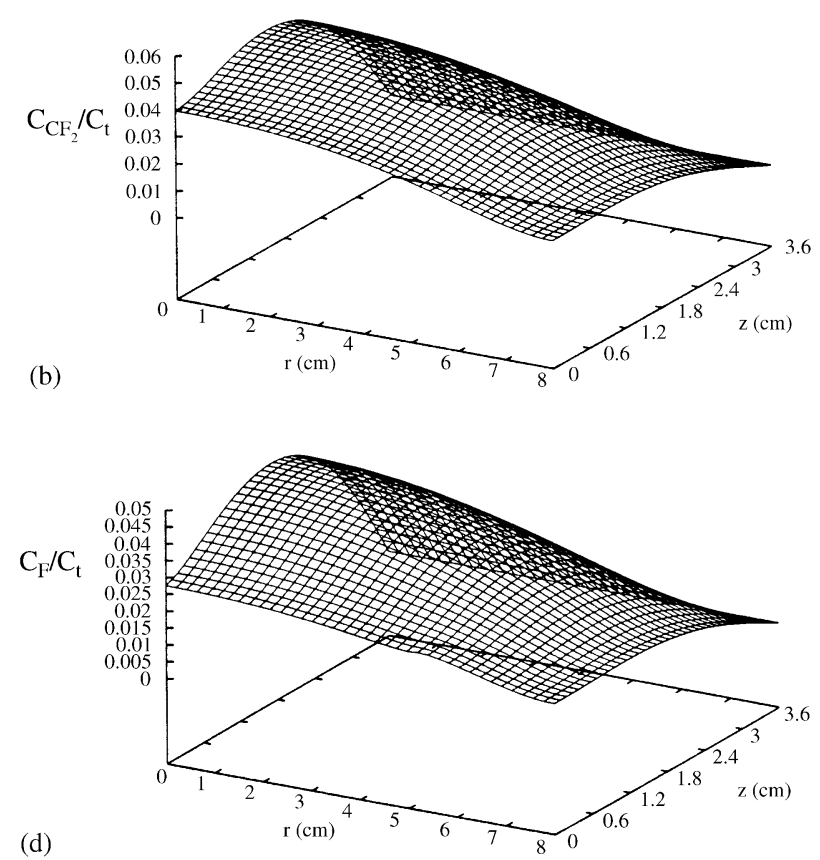

Fig. 2. Open-loop steady-state concentration profile of $\mathrm{CF}_{4}$ (a), $\mathrm{CF}_{2}$ (b), $\mathrm{CF}_{3}$ (c), $\mathrm{F}$ (d). 

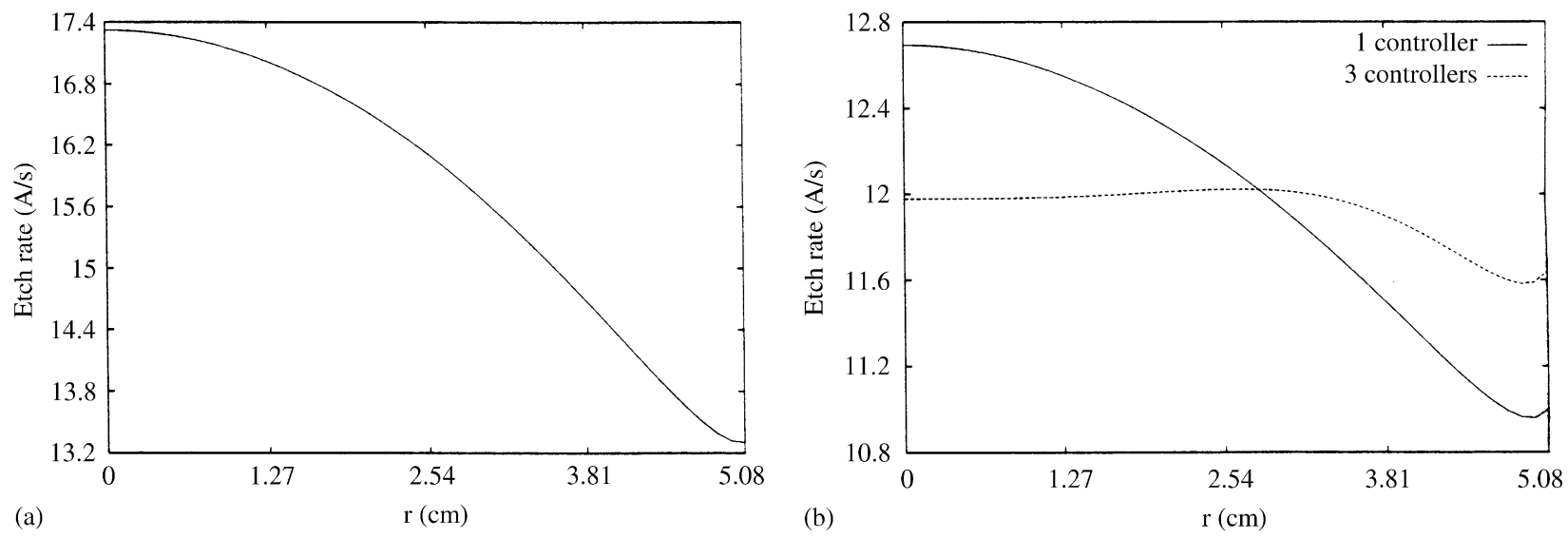

Fig. 3. Etching rate of $a$-Si as a function of wafer radius at final time for open-loop (a), closed-loop (b) process.

is mainly determined by the coverage of the surface by the absorbed $\mathrm{CF}_{3}$ and the concentration of $\mathrm{F}$ (which reacts towards $\mathrm{SiF}_{4}$ ), result in a nonuniform etching rate of the amorphous silicon profile along the radius of the wafer (Fig. 3a). We observe that the etching rate is maximum at the center of the wafer and decreases as the radius increases, matching the concentration profile of F (Fig. 2d). The small increase in the etching rate appearing close to the edge of the wafer is due to the concentration gradient of $\mathrm{F}$ in this location (Fig. 2d); this is a result of the fact that $\mathrm{F}$ does not react with the walls of the reactor (see also Park \& Economou (1991) for similar observations). A measure of the spatial nonuniformity, $R_{M}$, can be defined as $R_{M}=\left(R_{\max }-R_{\min }\right) / R_{\min }$, where $R_{\max }, R_{\min }$ are the maximum and minimum etching rates for $0 \mathrm{~cm} \leqslant r \leqslant 5.08 \mathrm{~cm}$. Using the values for $R_{\max }, R_{\min }$ from Fig. 3a, the nonuniformity measure for the openloop process is computed to be $R_{M}=30.2 \%$. This is a very large value, according to any industrial standard, which suggests the implementation of a feedback control system on the PE process to reduce radial film thickness nonuniformity.

\section{Controller design-closed-loop simulations}

In this section, we focus on the design and implementation of a feedback control system on the PE process in order to reduce the radial nonuniformity of the etching rate. The proposed control system utilizes point measurements of the etching rate at three locations across the radius of the wafer to manipulate the inlet concentration of $\mathrm{CF}_{4}$ in the showerhead and achieve uniform etching of a $500 \AA$ amorphous silicon thin film. In practice, a variety of on-line measurement techniques can be employed to determine the thickness of the $a$-Si film in situ (and thus, the etching rate), such as ellipsometry (Galarza, Khargonekar, \& Terry, 1999) and microwave conductivity (Neitzert, Hirsch, Kunst, \& Nell, 1995). The concentra- tion of $\mathrm{CF}_{4}$ at the showerhead was chosen as the manipulated variable because of its strong effect on the etching rate (this was verified with several open-loop simulation runs for different inlet $\mathrm{CF}_{4}$ concentrations; the effect of the inlet flow rate on the etching rate was also examined and found to be less effective (see Park and Economou (1991) for results on flow rate and etching rate dependency). Furthermore, the choice of the inlet $\mathrm{CF}_{4}$ concentration as manipulated variable was motivated by the realization that the suppression of the radial variation of the etching rate requires the use of a manipulated variable that is distributed across the radius of the reactor. We note that, in practice, the inlet carbon tetrafluoride concentration is manipulated by adjusting the composition of an inert gas such as $\mathrm{He}$ or $\mathrm{Ar}$ in the feed to the showerhead (the use of such a diluent does not affect the velocity profile inside the reactor, i.e., $v_{w}$ remains constant). Finally, the manipulation of the inlet $\mathrm{CF}_{4}$ concentration does not lead to perturbations (and possible jetting effects and instabilities) of the flow field when the controller is active, as it could happen in the case of using the flow rate in the showerhead as the manipulated variable.

Owing to the mildly nonlinear nature of the process model of Eq. (8) (it only includes bilinear nonlinearities since the assumption of isothermal operation allows considering the reaction rate parameters independent of temperature), a proportional integral (PI) controller with the following state-space description:

$\dot{\xi}=y_{s p}-y, \quad \xi(0)=0$,
$u=K\left(y_{s p}-y\right)+\frac{1}{\tau} \xi$,

was initially used to manipulate the inlet concentration of $\mathrm{CF}_{4}$ at the showerhead to achieve offsetless set-point tracking. The area of the showerhead in which the inlet concentration of $\mathrm{CF}_{4}$ is manipulated was assumed to be the circular area over the wafer, while in the region of the 


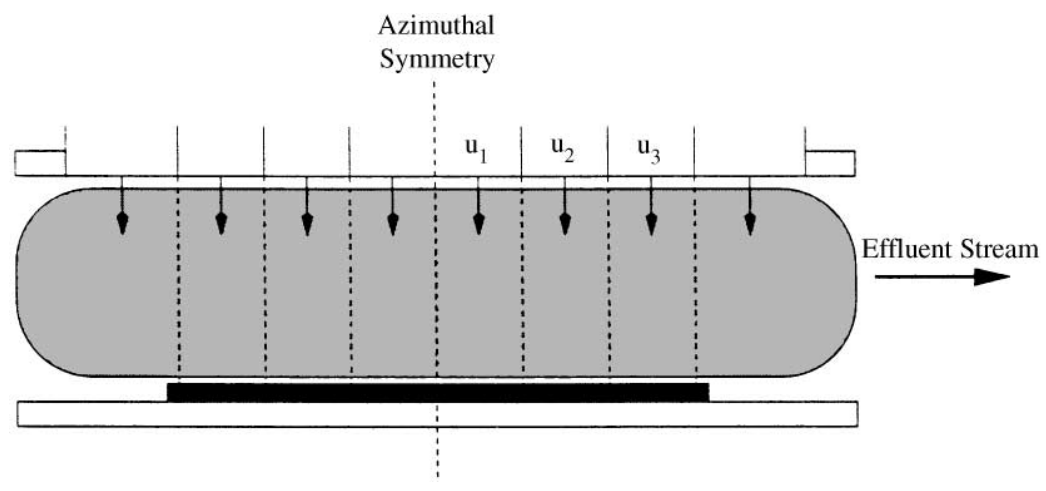

Fig. 4. Control configuration for PE reactor.

showerhead outside the wafer the feed was taken to be pure $\mathrm{CF}_{4}$ for all times. In the controller of Eq. (13), $\xi$ is the controller state variable, $y_{s p}$ denotes the setpoint value of the etching rate, $y$ denotes the on-line measurement of the etching rate, $K$ is the proportional controller gain and $\tau$ is the integral time constant. The manipulated input, $u$, was defined as $u=c_{w, 1} / c_{t}$, where $c_{w, 1}$ represents the concentration of $\mathrm{CF}_{4}$ at the showerhead, and the controlled output was defined as $y(t)=0.25 \rho_{\mathrm{Si}} k_{\mathrm{etch}} c_{4}\left(t, r_{w} /\right.$ $2,0)\left(1-\theta\left(t, r_{w} / 2\right)\right)$, where $r_{w}$ is the radius of the wafer, and was computed by using 1 measurement of the etching rate at radius $r=r_{w} / 2$. Note that since the etching rate setpoint $y_{s p}$ was set equal to $12.0 \AA / \mathrm{s}$, the time required for the etching of a $500 \AA$ thin film is approximately $42 \mathrm{~s}$ (the corresponding time for the open-loop process is $36 \mathrm{~s}$ ). Fig. $3 \mathrm{~b}$ shows the closed-loop etching rate as a function of wafer radius, while Fig. 5a shows the corresponding manipulated input profile (note that $u=0.0$ means that no $\mathrm{CF}_{4}$ enters the reactor, while $u=1.0$ means that the feed consists of pure $\mathrm{CF}_{4}$ ). The controller decreases the radial nonuniformity of the etching rate to $R_{M}=15.8 \%$ (note that in the open-loop system the nonuniformity is $R_{M}=30.2 \%$ ). Even though the use of a single PI controller reduces the film thickness nonuniformity compared to the one of the open-loop process, the reduction obtained is not significant enough, thereby motivating the design of a new feedback control system.

To further reduce the film thickness nonuniformity, we divide the showerhead area into three concentric regions (Fig. 4) and use three PI controllers to adjust the inlet $\mathrm{CF}_{4}$ concentration in each one of these regions. We note that no controller was used in the showerhead region outside of the wafer, and the feed was pure $\mathrm{CF}_{4}$. The idea for dividing the showerhead region into three concentric subregions is based on the realization that the effective control of a variable that is distributed in space (as is the case with the etching rate in this problem) should be achieved by independently controlled manipulated inputs that are also distributed in space. The state-space representation of the feedback control system then takes
Table 5

Parameters for the 3 PI controllers

\begin{tabular}{lllllll}
\hline$\kappa$ & $K_{\kappa}$ & $\tau_{\kappa}(\mathrm{s})$ & $r_{s, \kappa}(\mathrm{cm})$ & $r_{f, \kappa}(\mathrm{cm})$ & $r_{c, \kappa}(\mathrm{cm})$ & $y_{s p, \kappa}(\mathrm{A} / \mathrm{s})$ \\
\hline 1 & 0.1 & 5.0 & 0.00 & 2.00 & 1.00 & 12.0 \\
2 & 0.1 & 5.0 & 2.00 & 3.50 & 2.75 & 12.0 \\
3 & 0.1 & 5.0 & 3.50 & 5.08 & 4.00 & 12.0 \\
\hline
\end{tabular}

the form

$\dot{\xi}_{\kappa}=y_{s p, \kappa}-y_{\kappa}, \quad \xi_{\kappa}(0)=0, \quad \kappa=1,2,3$,

$u_{\kappa}=K_{\kappa}\left(y_{s p, \kappa}-y_{\kappa}\right)+\frac{1}{\tau_{\kappa}} \xi_{\kappa}$,

where $u_{\kappa}(t)$ is the $\kappa$ th control action and $r_{i, \kappa}$ and $r_{f, \kappa}$ are the internal and external radii of the region where the $\kappa$ th control action is applied. The $\kappa$ th controlled output for this region is defined as $y_{\kappa}(t)=0.25 \rho_{\mathrm{Si}} k_{\mathrm{etch}} c_{4}\left(t, r_{c, \kappa}, 0\right)$ $\left(1-\theta\left(t, r_{c, \kappa}\right)\right)$, where $r_{c, \kappa}$ is the position of the $\kappa$ th on-line measurement. Table 5 provides the parameters and set-point values for the three PI controllers, and the radii of the regions where they are applied.

Fig. $3 \mathrm{~b}$ shows the closed-loop etching rate in the case of using three PI controllers, while Fig. 5b displays the corresponding manipulated input profiles. It is clear that the use of three spatially distributed PI controllers has substantially reduced the film thickness nonuniformity; the nonuniformity measure for the profile of Fig. 5 is $R_{M}=3.8 \%$ which is much less than $30.2 \%$ (uncontrolled process) and $15.8 \%$ (single PI controller). From the above closed-loop system simulation results, it is evident that the division of the showerhead region into three concentric subregions is the main reason for the significant reduction in etching rate nonuniformity.

\section{Conclusions}

This work focused on the implementation of a feedback control system on a parallel electrode PE process 

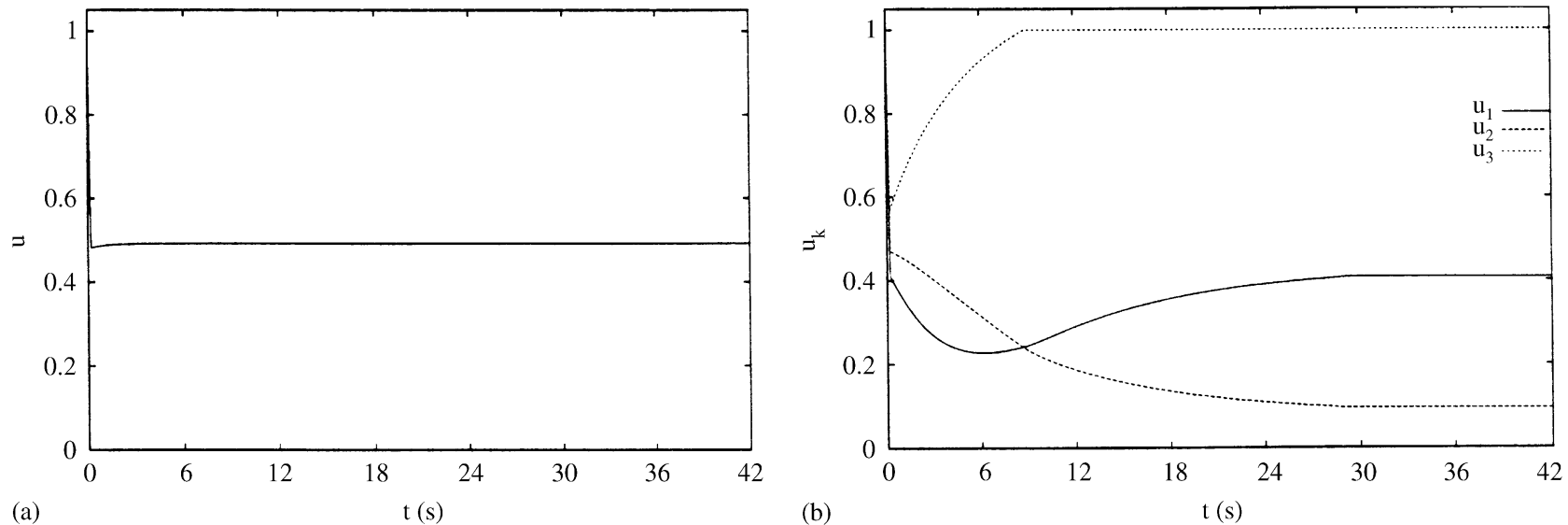

Fig. 5. Manipulated input profiles for the case of using one PI controller (a) and three PI controllers (b).

with showerhead arrangement used to etch a $500 \AA$ amorphous silicon thin film on a 4 in wafer. The feedback control system consists of three spatially distributed proportional integral controllers that use measurements of the etching rate at three locations across the wafer, to manipulate the inlet concentration of carbon tetrafluoride in the showerhead and achieve a uniform etching rate across the wafer. The controller was implemented on a detailed fundamental model of the process which accounts for diffusive and convective mass transfer, bulk and surface reactions, and nonuniform fluid flow and plasma electron density profiles. The implementation of the proposed control system was shown to reduce the etching rate nonuniformity from 30.2 to $3.8 \%$.

\section{Acknowledgements}

Financial support from a National Science Foundation CAREER award, CTS-9733509, is gratefully acknowledged.

\section{References}

Armaou, A., \& Christofides, P. D. (1999). Plasma-enhanced chemical vapor deposition: Modeling and control. Chemical Engineering Science, 54, 3305-3314.

Belenguer, P., \& Boeuf, J. P. (1990). Transition between different regimes of RF glow discharges. Physical Review A, 41, 4447-4459.

Bushman, S., Edgar, T., \& Trachtenberg, I. (1997). Modeling of plasma etch systems using ordinary least squares, recurrent neural network, and projection to latent structure models. Journal of the Electrochemical Society, 144, 1379-1389.

Butler, S. W., McLaughlin, K. J., Edgar, T. F., \& Trachtenberg, I. (1991). Development of techniques for real-time monitoring and control in plasma etching II. multivariable control system analysis of manipulated, measured and performance variables. Journal of the Electrochemical Society, 138, 2727-2735.
Economou, D. J., Park, S., \& Williams, G. D. (1989). Uniformity of etching in parallel plate plasma reactors. Journal of the Electrochemical Society, 136, 188-198.

Edelson, D., \& Flamm, D. (1984). Computer simulation of a $\mathrm{CF}_{4}$ plasma etching silicon. Journal of Applied Physics, 56, 1522-1531.

Flamm, D., Donnelly, V., \& Ibbotson, D. (1983). Basic chemistry and mechanisms of plasma etching. Semiconductor International, 6, 136-143.

Flamm, D., Donnelly, V., \& Mucha, J. (1981). The reaction of fluorine atoms with silicon. Journal of Applied Physics, 52, 3633-3639.

Galarza, C., Khargonekar, P., \& Terry, F. J. (1999). Real-time estimation of patterned wafer parameters using in situ spectroscopic ellipsometry. In Proceedings of the IEEE International Conference on Control Applications, Kohala Coast, HI, (pp. 773-778).

Hankinson, M., Vincent, T., Irani, K., \& Khargonekar, P. (1997). Combined real-time and run-to-run control of etch depth and spatial uniformity in plasma etching. Journal of the Electrochemical Society, 144, 2473-2479.

McLaughlin, K. J., Butler, S. W., Edgar, T. F., \& Trachtenberg, I. (1991). Development of techniques for real-time monitoring and control in plasma etching I. Response surface modeling of $\mathrm{CF}_{4} / \mathrm{O}_{2}$ and $\mathrm{CF}_{4} / \mathrm{H}_{2}$ etching of silicon and silicon dioxide. Journal of the Electrochemical Society, 138, 789-799.

Mozumder, P. K., Saxena, S., \& Collins, D. J. (1994). A monitor wafer based controller for semiconductor processes. IEEE Transactions on Semiconductor Manufacturing, 7, 400-411.

Neitzert, H. C., Hirsch, W., Kunst, M., \& Nell, M. E. A. (1995). In situ thickness control during plasma deposition of hydrogenated amorphous silicon films by time-resolved microwave conductivity measurements. Applied Optics, 34, 676-680.

Park, S., \& Economou, D. J. (1990). Numerical simulation of a singlewafer isothermal plasma etching reactor. Journal of the Electrochemical Society, 137, 2624-2634.

Park, S., \& Economou, D. J. (1991). A mathematical model for etching of silicon using $\mathrm{CF}_{4}$ in a radial flow plasma reactor. Journal of the Electrochemical Society, 138, 1499-1508.

Plumb, I., \& Ryan, K. (1986). A model of the chemical processes occurring in $\mathrm{CF}_{4} / \mathrm{O}_{2}$ discharges used in plasma etching. Plasma Chemistry and Plasma Processing, 6, 205-230.

Reid, R. C., Prausnitz, J. M., \& Poling, B. E. (1987). The Properties of Gases and Liquids. (4th ed.). New York: McGraw-Hill.

Ryan, K., \& Plumb, I. (1986). A model for the etching of $\mathrm{Si}$ in $\mathrm{CF}_{4}$ plasmas: Comparison with experimental measurements. Plasma Chemistry and Plasma Processing, 6, 231-246. 
Van Antwerp, J., Braatz, R., \& Sahinidis, N. (1997). Robust nonlinear control of plasma etching. In Proceedings of the symposium on silicon nitride and silicon dioxide thin insulating films, Montreal, Canada, (pp. 454-462).
Venkatesan, S., Trachtenberg, I., \& Edgar, T. (1987). Effect of flow direction on etch uniformity in parallel-plate (radial flow) isothermal plasma reactors. Journal of the Electrochemical Society, 134, 3194-3197. 